\title{
Development of a data-driven model for turbulent heat transfer in turbomachinery
}

\author{
Francesco Aldo Tucci*, Giovanni Delibra, and Alessandro Corsini \\ Department of Mechanical and Aerospace Engineering, Sapienza University of Rome, Via \\ Eudossiana 18, 00184 Rome, Italy
}

\begin{abstract}
Machine Learning (ML) algorithms have become popular in many fields, including applications related to turbomachinery and heat transfer. The key properties of ML are the capability to partially tackle the problem of slowing down of Moore's law and to dig-out correlations within large datasets like those available on turbomachinery. Data come from experiments and simulations with different degree of accuracy, according to the test-rig or the CFD approach. When dealing with modelling of turbulent flows in turbomachinery there is a constant trade-off between accuracy and computational costs, but starting from the large amount of data on turbomachinery performance, with $\mathrm{ML}$ it is possible to train a learner to correct and improve CFD. The aim of this work is to investigate an innovative data-driven approach that could lead to a significant improvement in the analysis of heat transfer in turbulent flows. The effects of Reynolds number and wall temperature on heat transfer for a double forward-facing step with two squared obstacles were investigated by numerical simulations carried out in OpenFOAM. Then a machine-learnt model was derived using a regression algorithm. The results of regressor showed that a data-driven approach can effectively predict results of the RANS model.
\end{abstract}

\section{Introduction}

Modelling of heat transfer is a key issue in many engineering applications, especially when dealing with Computational Fluid Dynamics (CFD) of turbulent flows with heat transfer. Among many open issues, most comes from the Boussinesq approximation and in particular to the limit of having Reynolds fluxes aligned to Reynolds stresses [1]. This condition is nonphysical, especially in strongly non-isotropic turbulence regions and in particular along solid walls. Moreover, these regions are also those which pose the most challenging conditions when dealing with grid refinement for CFD due to the resolution needed to integrate NavierStokes to the wall [2], and unfortunately most industrial CFD design and optimization loops still rely on the use of wall functions to model the near-wall flow and thermal fields.

Since wall functions are usually calibrated on simple attached-flows conditions, this eventually leads to increase of errors when solving more complex flows with impingement, recirculation, reattachment, transition and other phenomena.

\footnotetext{
*Corresponding author: francescoaldo.tucci@uniroma1.it
} 
In the following we discuss the possibility of deriving the distributions of turbulent viscosity and diffusivity in a low-Reynolds RANS approach using machine learning, and in particular to apply this strategy to a complex flow configuration with multiple flow features, such as geometry-induced separation, impingement, reattachment, wakes using data from multiple Reynolds numbers. In particular, the case here reproduced is the double forwarding facing step with squared obstacles previously investigated in [3]. The case here considered is the one with the configuration: $\mathrm{L}=1.6 \mathrm{~m}, \mathrm{a}=1 \mathrm{~m}, \mathrm{~b}=0.2 \mathrm{~m}, \mathrm{c}=0.4 \mathrm{~m}, \mathrm{H}=0.1 \mathrm{~m}, \mathrm{~h} 1=\mathrm{h} 2=0.02 \mathrm{~m}$, $\mathrm{w}=\mathrm{t}=0.01 \mathrm{~m}$, Figure 1 . Combinations of Reynolds number and boundary conditions for temperature will be discussed in section 2.2 and is summarized in Table 1.
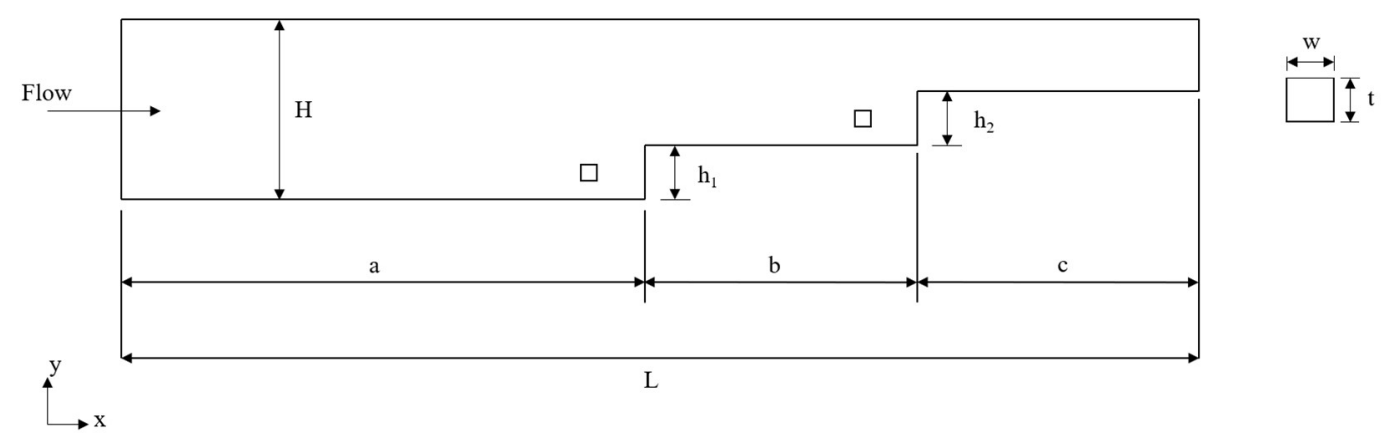

Figure 1 - Sketch of the computational domain [3]

This is a preliminary work to understand the feasibility of such approach in a simplified 2D geometry with multiple flow regimens at different Reynolds.

\section{Methodology}

\subsection{CFD method}

Computations were carried out using the OpenFOAM v1812 [4] using the buoyantBoussinesqSimpleFoam solver for incompressible flows with heat transfer. In this case heat transfer is calculated assuming temperature to be a transported scalar with Prandtl number $\operatorname{Pr}=0.71$ [5] and turbulence closure relied on the Launder-Sharma k- $\varepsilon$ model [6]. The linearized system of equations was solved using conjugate gradient solver for all quantities. Tolerance was set to $10^{-5}$ for pressure and $10^{-8}$ for all the other equations. Numerical schemes used entailed central differencing for gradients, QUICK for divergence terms and upwind for Laplacian terms. Boundary conditions entailed mass flow rate at the inlet with $\mathrm{TI}=5 \%$, $v_{\tau} / v=60, T=293 \mathrm{~K}$. On solid surfaces no-slip conditions were used with integration to the wall approach and an average value of $\mathrm{y}^{+}$equal to 0.88 for the highest Reynolds number considered (maximum $\mathrm{y}^{+}=1.1$ in this case). Fixed temperature was imposed on heated walls according to the conditions investigated in Table 1. At the outlet convective conditions were imposed. The computational domain is $2 \mathrm{D}$ and the final mesh entails 622,400 cells. Convergence of grid was investigated using Nusselt number on the heated walls as convergence parameters using 300,200, 622,400 and 1,120,000 cells. The difference in average Nusselt is below $1 \%$ between 622,400 and 1,120,000 cells. Results of the 9 computations were used as training and testing data for the ML approach. 


\subsection{Dataset}

In the present work, the regression algorithm is designed to implement a data-driven approach for heat-transfer in turbulent flows, in particular the final aim is to predict the turbulent thermal diffusivity $\left(\alpha_{t}\right)$ and turbulent viscosity $\left(v_{t}\right)$ fields.

The overall training dataset of the regression algorithm consists of $\sim 95 \mathrm{M}$ elements and is the set of nine different smaller datasets each of $\sim 10.5 \mathrm{M}$ elements covering three different Reynolds numbers $\left(\mathrm{Re}_{1}=30000, \mathrm{Re}_{2}=80000\right.$ and $\left.\mathrm{Re}_{3}=100000\right)$ and three different temperature values $\left(T_{1}=313 \mathrm{~K}, \mathrm{~T}_{2}=423 \mathrm{~K}\right.$ and $\left.\mathrm{T}_{3}=626 \mathrm{~K}\right)$. Table 1 gives a summary of the nine different cases.

Table 1 - Training Case Matrix

\begin{tabular}{c|ccc}
\hline & $\mathbf{R e}_{\mathbf{1}}=\mathbf{3 0 0 0 0}$ & $\mathbf{R e}_{2}=\mathbf{8 0 0 0 0}$ & $\mathbf{R e}_{3}=\mathbf{1 0 0 0 0 0}$ \\
\cline { 2 - 4 } $\mathbf{T}_{\mathbf{1}}=\mathbf{3 1 3 K}$ & case 1 & case 4 & case 7 \\
$\mathbf{T}_{\mathbf{2}}=\mathbf{4 2 3 K}$ & case 2 & case 5 & case 8 \\
$\mathbf{T}_{\mathbf{3}}=\mathbf{6 2 6 K}$ & case 3 & case 6 & case 9 \\
\hline
\end{tabular}

\subsection{Feature creation and normalization}

Table 2 - Feature Creation \& Normalization

\begin{tabular}{|c|c|c|c|}
\hline Variable & Label & Definition & $\mathbf{N}$ \\
\hline Velocity magnitude & $\operatorname{mag}(\mathrm{U})$ & $\|\mathrm{U}\|$ & - \\
\hline Temperature & $\mathrm{T}$ & $\mathrm{T}$ & - \\
\hline Dissipation rate of $k$ & epsilon & $\varepsilon$ & $\mathrm{U}^{3}{ }_{\text {ref }} / \mathrm{L}_{\mathrm{ref}}$ \\
\hline Pressure & $\mathrm{p}$ & $\mathrm{p}$ & $\mathrm{U}_{\text {ref }}^{2}$ \\
\hline Turbulent kinetic energy & $\mathrm{k}$ & $\mathrm{k}$ & $\mathrm{U}^{2}{ }_{\mathrm{ref}}$ \\
\hline $\begin{array}{l}\text { Angle between velocity and } \\
\text { pressure gradient }\end{array}$ & teta & $\theta$ & - \\
\hline $\begin{array}{l}\text { Angle between velocity and } \\
\text { temperature gradient }\end{array}$ & beta & $\beta$ & - \\
\hline $\begin{array}{l}\text { Angle between pressure and } \\
\text { temperature gradients }\end{array}$ & zeta & $\zeta$ & - \\
\hline Eddy diffusivity & alphat & $\alpha_{t}$ & $\mathrm{U}_{\text {ref }} \cdot \mathrm{L}_{\text {ref }}$ \\
\hline Eddy viscosity & nut & $v_{t}$ & $\mathrm{U}_{\text {ref }} \cdot \mathrm{L}_{\text {ref }}$ \\
\hline Magnitude of strain tensor & Sii & $\sum_{\mathrm{ij}}\left(\partial \mathrm{U}_{\mathrm{ij}} / \partial \mathrm{x}_{\mathrm{j}}+\partial \mathrm{U}_{\mathrm{ij}} / \partial \mathrm{x}_{\mathrm{i}}\right)$ & $\mathrm{U}_{\text {ref }} / \mathrm{L}_{\mathrm{ref}}$ \\
\hline Magnitude of rotation tensor & $\mathrm{W}_{\mathrm{ii}}$ & $\sum_{\mathrm{ij}}\left(\partial \mathrm{U}_{\mathrm{ij}} / \partial \mathrm{x}_{\mathrm{j}}-\partial \mathrm{U}_{\mathrm{ij}} / \partial \mathrm{x}_{\mathrm{i}}\right)$ & $\mathrm{U}_{\mathrm{ref}} / \mathrm{L}_{\mathrm{ref}}$ \\
\hline Pressure gradient & $\nabla p$ & $\sum_{\mathrm{i}}(\partial \mathrm{p} / \partial \mathrm{xi})$ & $\mathrm{U}^{2}{ }_{\text {ref }} / \mathrm{L}_{\text {ref }}$ \\
\hline Gradient of $\varepsilon$ & $\nabla \varepsilon$ & $\sum_{\mathrm{i}}(\partial \varepsilon / \partial \mathrm{xi})$ & $\mathrm{U}^{3}{ }_{\text {ref }} / \mathrm{L}_{\text {ref }}^{3}$ \\
\hline Eddi viscosity gradient & $\nabla v_{\mathrm{t}}$ & $\sum_{\mathrm{i}}\left(\partial v_{\mathrm{t}} / \partial \mathrm{xi}\right)$ & $\mathrm{U}_{\mathrm{ref}}$ \\
\hline Gradient of k & $\nabla \mathrm{k}$ & $\sum_{\mathrm{i}}(\partial \mathrm{k} / \partial \mathrm{xi})$ & $\mathrm{U}^{2}{ }_{\text {ref }} / \mathrm{L}_{\mathrm{ref}}$ \\
\hline Temperature gradient & $\nabla \mathrm{T}$ & $\sum_{\mathrm{i}}(\partial \mathrm{T} / \partial \mathrm{xi})$ & $1 / \mathrm{L}_{\mathrm{ref}}$ \\
\hline
\end{tabular}

The training datasets initially includes information on seventeen features (Table 2). To avoid problems related to the frame of references all the selected features are scalars. In [7], Goodfellow et al. stated that ML algorithms are negatively affected by features that exhibit large variations in magnitude, in fact the predictor could overestimate the influence of features with the smallest values. To avoid this, features were normalized following the findings of [8] that pointed out how this method is more suitable than usual normalization 
techniques used in ML and was positively tested in [9]-[11]. According to [8], most features were normalized as:

$$
f^{\prime}=\frac{f}{|f|+|N|}
$$

where $f^{\prime}$ is the normalized feature, $f$ the original feature, and $N$ a normalization factor summarized in Table 2, where $U_{\text {ref }}=\operatorname{mag}(U)$ and $L_{\text {ref }}=4 \cdot \operatorname{mag}(U) / S_{\text {ii }}$ are respectively the reference velocity and length. This normalization, unlike most ML techniques, is based on local flow and thermal field properties. All the features that don't show a value for $\mathrm{N}$ in Table 2 were normalized with minMax scaler [7] as a local normalization would result in a constant value (like for velocity) or would not be suitable (like for angles).

\subsection{Feature selection}

A valid predictive model must guarantee that there is no obvious predominance between the input features and the output. Moreover, ML algorithms do not satisfactory work with data that present a strong direct correlation between each other since this would be transferred directly to the final model.

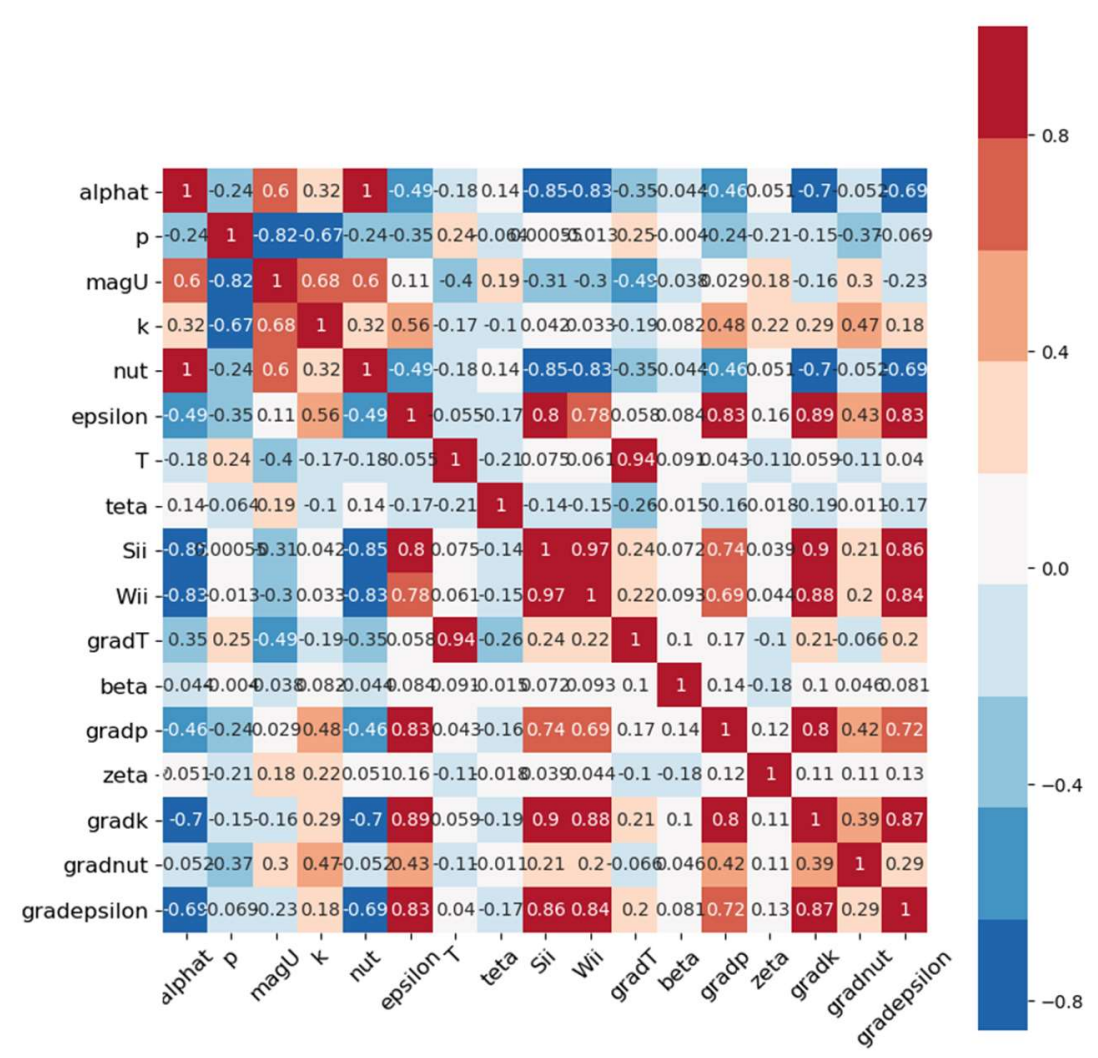

Figure 2 - Correlation matrix on training data.

In so doing, an Exploratory Data Analysis (EDA) is performed to verify the accuracy of the former feature selection and a correlation analysis of the data is reported in Figure 2. 
From the correlation matrix it is possible to observe that the strain tensor $\left(\mathrm{S}_{\mathrm{ii}}\right)$ and the rotation tensor $\left(\mathrm{W}_{\mathrm{ii}}\right)$ are highly correlated and that $\beta, \theta$ and $\zeta$ are not correlated to $v_{\mathrm{t}}$ or $\alpha_{\mathrm{t}}$. Thus, we decide to not include $\mathrm{W}_{\mathrm{ii}}, \beta, \theta$ and $\zeta$ in the training data.

\subsection{Regression algorithm and training}

XGBoost [12] was selected as regression algorithm due to its resilience in working with continuous data with a high number of outliers [12]. Objective of the training was set to linear regression, with subsampling of columns set to $30 \%$, learning rate to 0.1 , maximum depth of each tree to 50 , number of trees to 200 . These hyperparameters were optimized using a random search algorithm [13].

The general framework is made up of two phases: i) training and test and ii) prediction. During training, the individual datasets relating to the nine cases are grouped and crossed based on the Reynolds Number of references to build three different training datasets (Table 3).

Table 3 - Training Datasets

\begin{tabular}{cc}
\hline Training Dataset & Reynolds Numbers \\
\hline \multirow{2}{*}{ Dataset A } & $\mathrm{Re}=30000$ \\
& $\mathrm{Re}=80000$ \\
\hline \multirow{2}{*}{ Dataset $\mathrm{B}$} & $\mathrm{Re}=30000$ \\
& $\mathrm{Re}=100000$ \\
\hline \multirow{2}{*}{ Dataset C } & $\mathrm{Re}=80000$ \\
& $\mathrm{Re}=100000$ \\
\hline
\end{tabular}

Each of these three datasets is used to train a regression model for $\alpha_{t}$ and $v_{t}$. A logarithmic transformation is performed on $\alpha_{\mathrm{t}}$ and $v_{\mathrm{t}}$ which are strongly inclined variables in a more normalized dataset. The use of the logarithm of these two variables improves the fit of the regression model by transforming the distribution of the features into a bell curve of a more normal shape. Figure 3 shows the values of $\alpha_{t}$ and $v_{t}$ predicted from the models with respect to the training data. For each of the six training phases root-mean-square error (RMSE) remains below of $1 \%$, this means that the training is successfully achieved and verifies the initial assumptions on feature selection and the framework architecture. As an example, Figure 4 shows the field of $\alpha_{t}$ relating to case 3 , which perfectly reproduces the real values.

\section{Results}

Prediction follows training of the algorithm: to test the generalization of the models the regression model is forwarded to the three cases not included in the training datasets. Cross validation is required to evaluate the generality of the models out of the training data, the target is to find out how the model is performing based on training data. 
E3S Web of Conferences 197, 11006 (2020)

https://doi.org/10.1051/e3sconf/202019711006

$75^{\circ}$ National ATI Congress
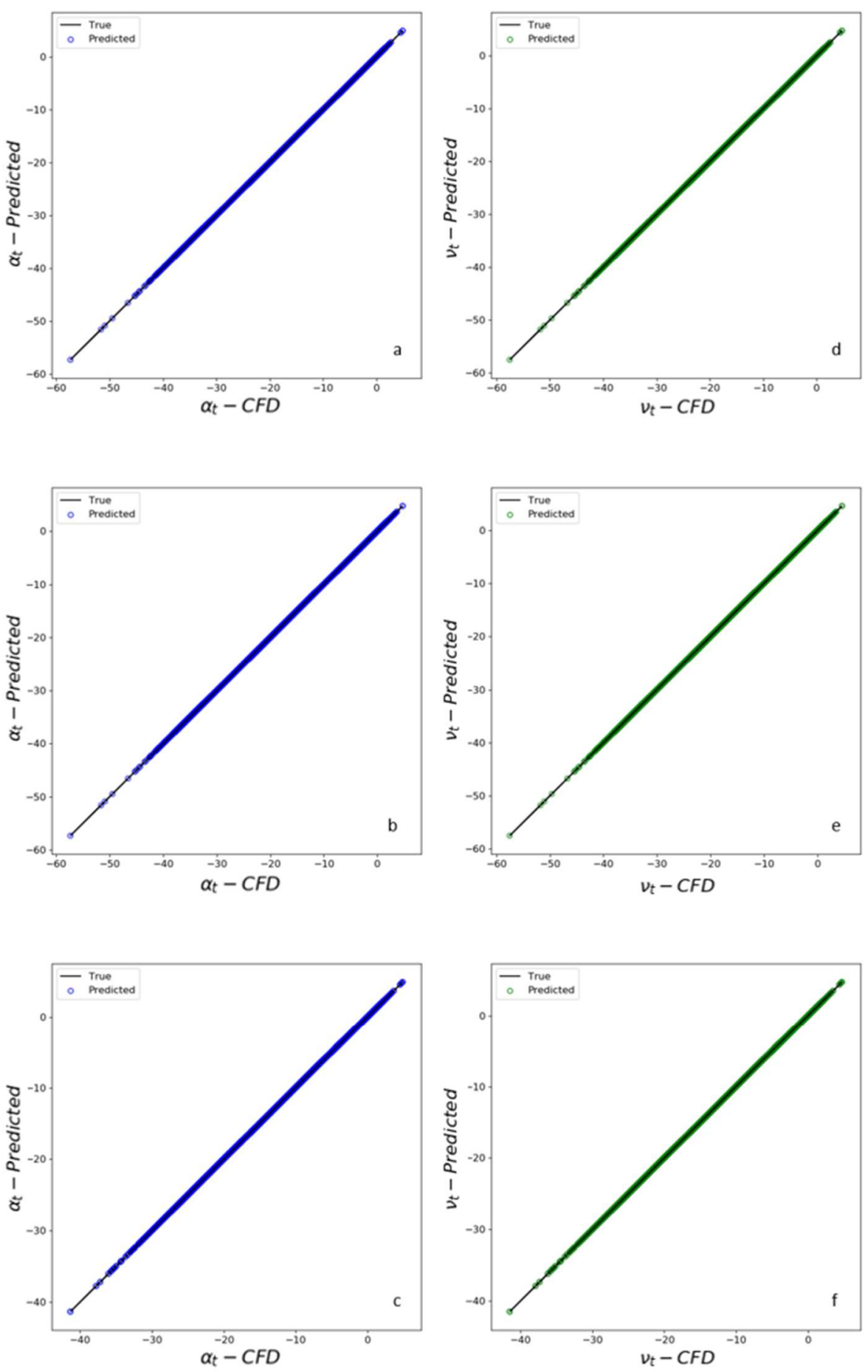

Figure 3 - Values of $\alpha_{t}$ and $v_{t}$ after the training phase: a) $\alpha_{t}$ - Dataset A, b) $\alpha_{t}$ - Dataset B, c) $\alpha_{t}-$ Dataset C, d) $v_{t}-$ Dataset A, e) $v_{t}-$ Dataset B, f) $v_{t}-$ Dataset C.

6 
By means of an interpolation process from Dataset B it is possible to predict both $\alpha_{\mathrm{t}}$ and $v_{t}$ fields of the three missing cases (case4-6) corresponding to Re 80000; similarly, with two extrapolation processes from Dataset A it is possible to predict both $\alpha_{t}$ and $v_{t}$ fields of the three missing cases (case 7-9) corresponding to Re 100000 and from Dataset $\mathrm{C}$ it is possible to predict that fields of the three missing cases (case1-3) corresponding to $\operatorname{Re} 30000$.

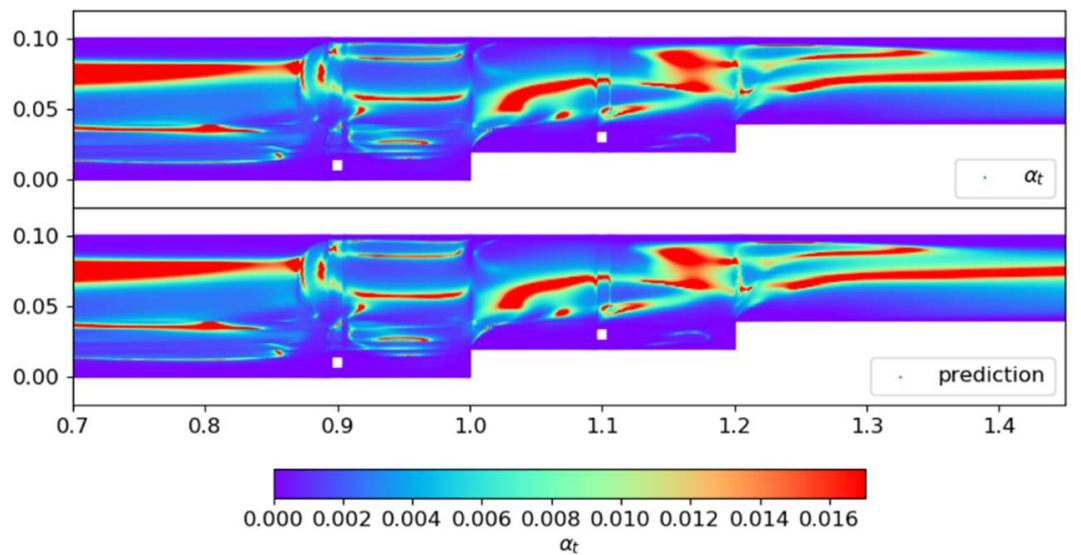

Figure 4 - Predicted $\alpha_{t}$ values with respect to training data (case 3 ).

The results of the interpolation process are highlighted in in Figure 5 and in Figure 6, where it is shown the good agreement of the predicted values of $\alpha_{t}$ and $v_{t}$ with their respective real values. For what concerns the extrapolation processes Figure 7 shows the prediction of $v_{t}$ relating to case 9 with a not perfect match with respect real values, Figure 8 depicts the prediction of $\alpha_{t}$ relating to case 3 with a match with respect real values even worse than the previous one.

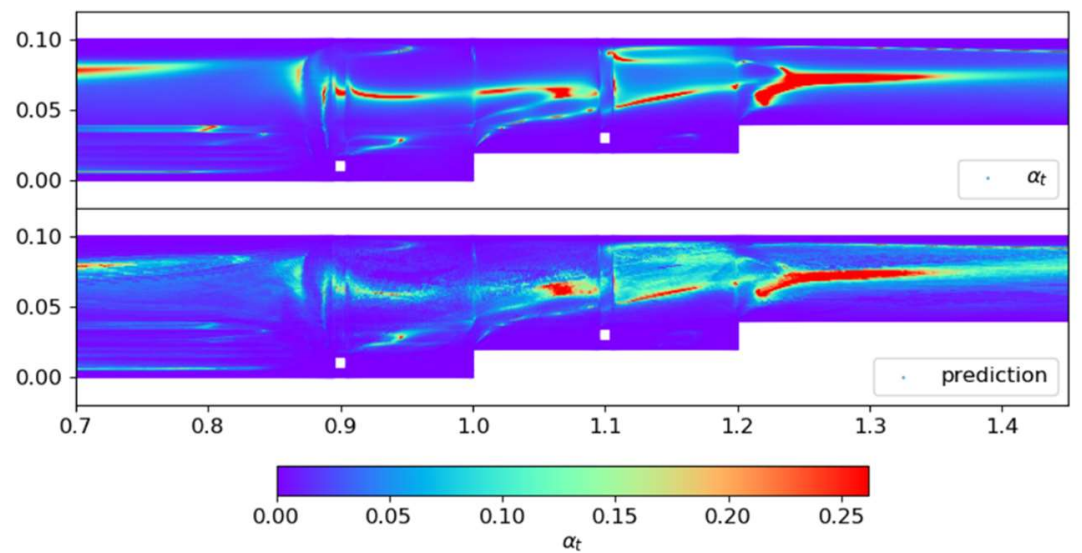

Figure 5 - Predicted $\alpha_{\mathrm{t}}$ values from interpolation process (case 6). 


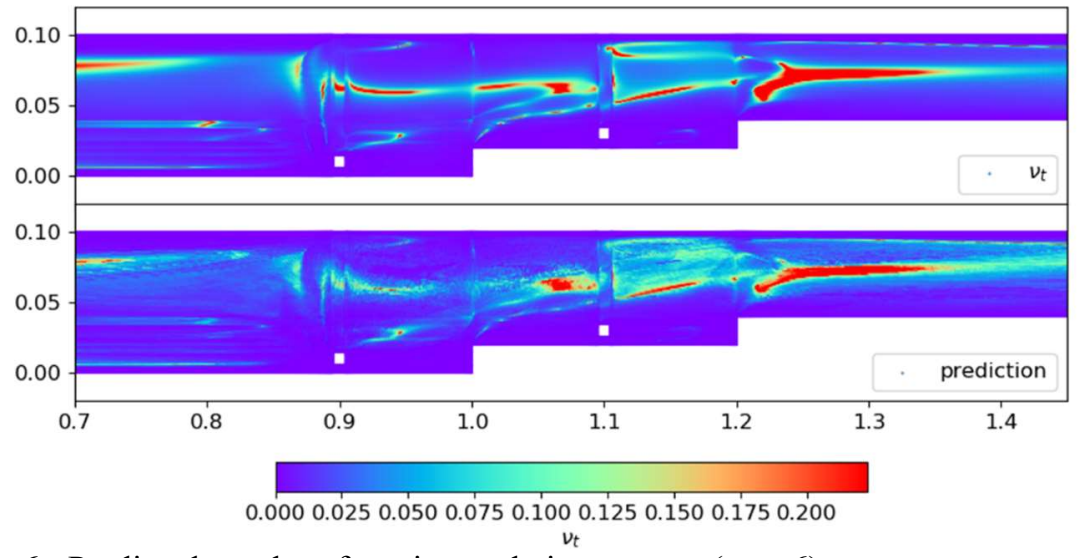

Figure 6 - Predicted $v_{\mathrm{t}}$ values from interpolation process (case 6).

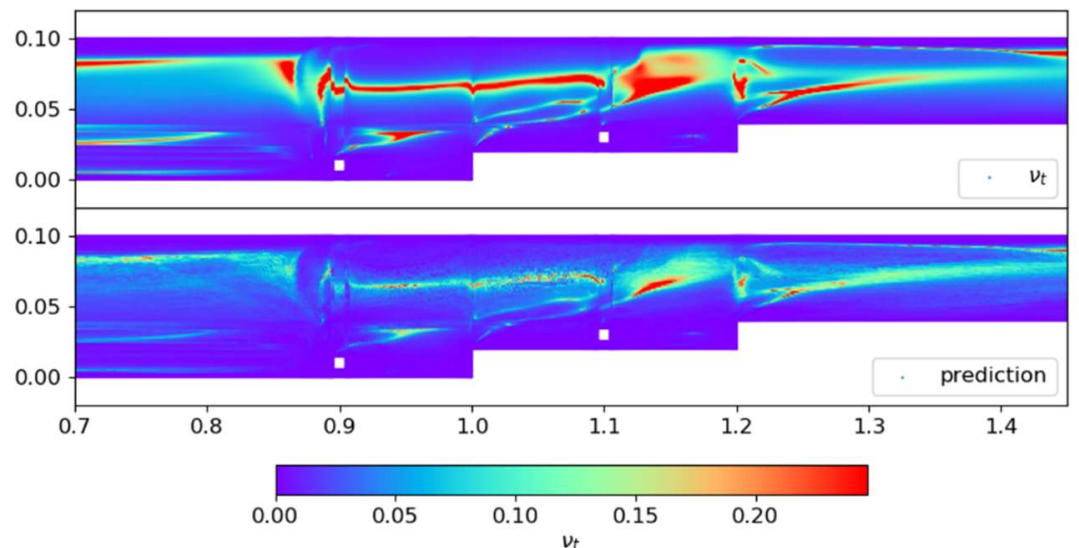

Figure 7 - Predicted $v_{\mathrm{t}}$ values from extrapolation process (case 9).

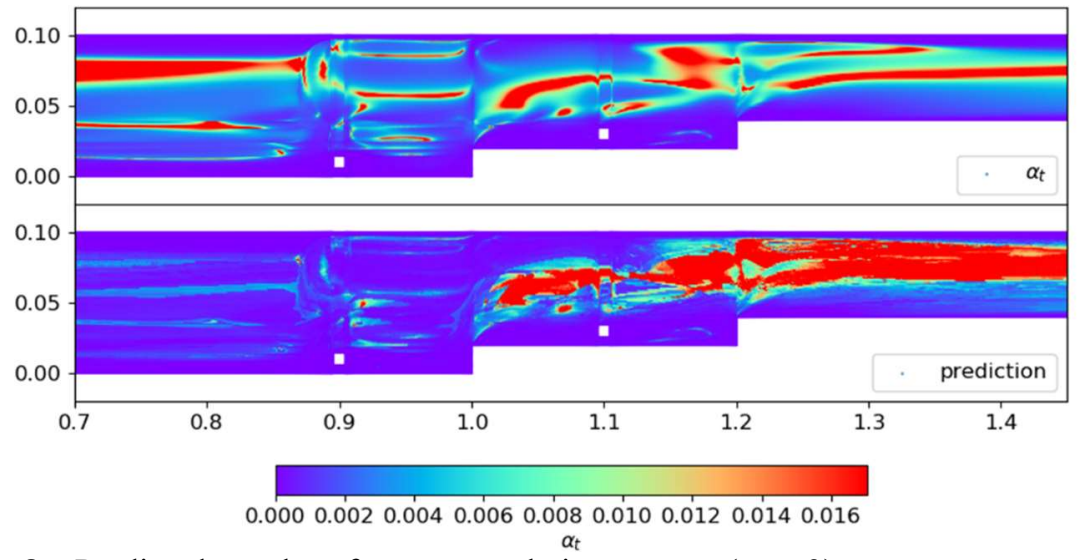

Figure 8 - Predicted $\alpha_{t}$ values from extrapolation process (case 3). 

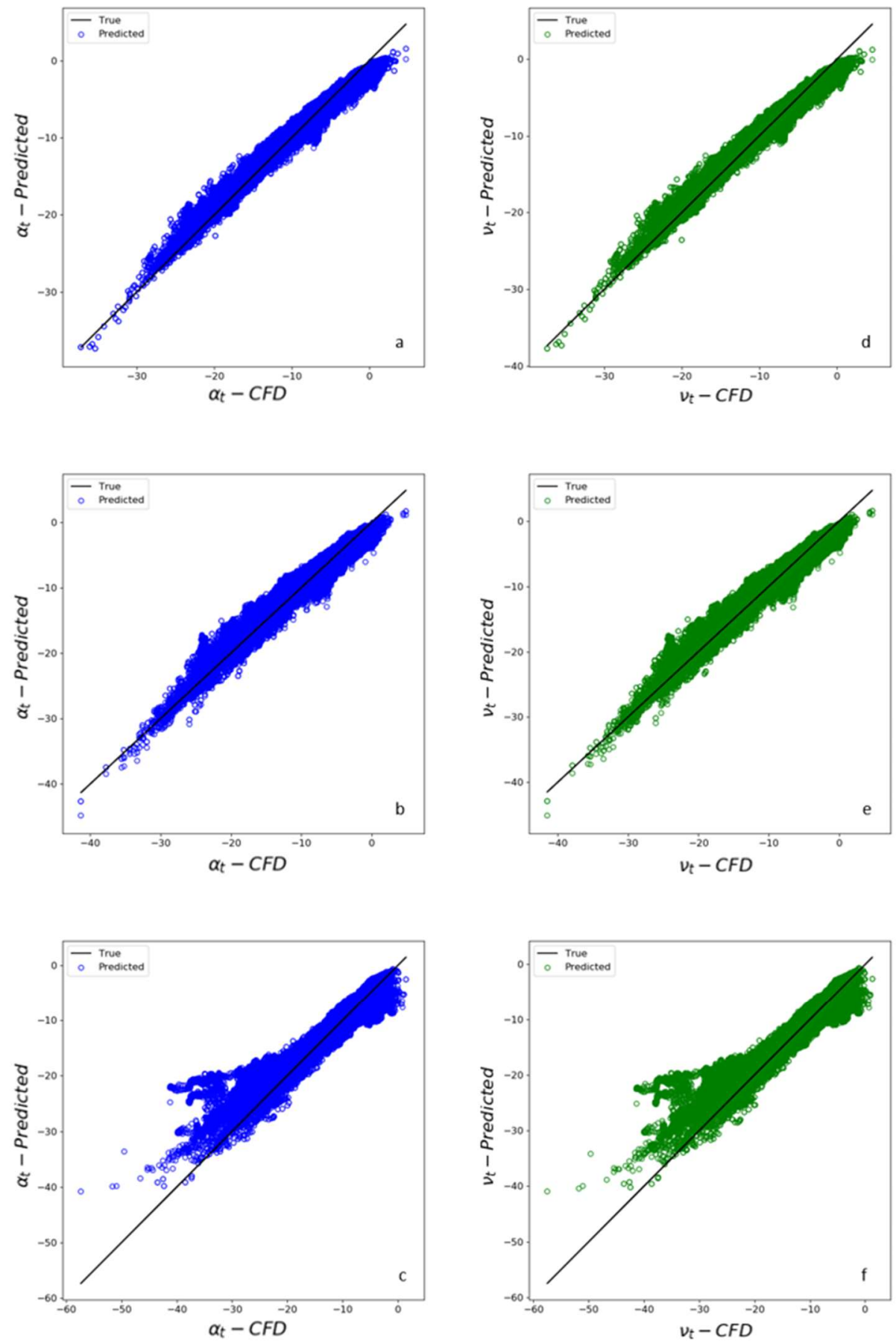

Figure 9 - Predicted values of $\alpha_{t}$ and $v_{t}$ : a) predicted $\alpha_{t}$ from Dataset A, b) predicted $\alpha_{t}$ from Dataset B, c) predicted $\alpha_{t}$ from Dataset C, d) predicted $v_{t}$ from Dataset A, e) predicted $v_{t}$ from Dataset $\mathrm{B}, \mathrm{f}$ ) predicted $v_{\mathrm{t}}$ from Dataset $\mathrm{C}$. 
Figure 9 shows the results of the regression models for both features $\alpha_{t}$ and $v_{t}$. It is possible to state that the models have good agreement with the CFD values in particular for the interpolation process, but when dealing with the extrapolation processes the predicted values deviate more from the real ones. Table 4 gives a summary of the root-mean-square error of predictions.

Table 4 - RMSE in cross-validation

\begin{tabular}{ccc}
\hline Training Dataset & RMSE $-\boldsymbol{\alpha}_{\mathbf{t}}$ & $\mathbf{R M S E}-\mathbf{v}_{\mathbf{t}}$ \\
\hline Dataset A & 0.79 & 0.79 \\
Dataset B & 0.83 & 0.84 \\
Dataset C & 1.68 & 1.69 \\
\hline
\end{tabular}

\section{Conclusions}

We investigated the possibility of predicting a more accurate distribution of eddy viscosity and eddy diffusivity in a RANS framework to possibly extend the characterization of a turbulent and thermal boundary layer to a more accurate wall function.

A dataset of 9 cases was created using low-Reynolds computations of a complex 2D test case characterized by many flow regimens, varying two parameters: Reynolds number and wall temperature.

Then, the complete set of data was characterized using correlation matrix and other EDA techniques to select the appropriate features to be used for regression. From a number of 17 original features only 13 were used.

A XGBoost regressor was then trained and tested. In so doing a series of sub-dataset were selected aiming at investigating the capability of the model of interpolating and extrapolating the correct fields of eddy viscosity and diffusivity.

Interpolation lead to good results, while extrapolation showed the limits of this approach, suggesting that maybe a more complex ML approach should be used.

From the response of the algorithm we can infer that interpolation could increase its accuracy increasing the number of Reynolds numbers used for the training of the algorithm as the regressor has a linear trend whilst in the considered range of Reynolds the flow characteristics have increasing turbulent behaviour without reaching a stable range of operations.

Nevertheless since this is also a preliminary work to assess the possibility of using lowReynolds approach to better tune an high-Reynolds wall function, we can see from Figures 4-8 that in the boundary layer the regressor has a high accuracy and therefore it is possible to use these data to tune a dynamic wall function that takes into account the local properties of the flow and thermal fields.

\section{References}

[1]. Delibra, G., Borello, D., Hanjalic, K., Rispoli, F., An les insight into convective mechanism of heat transfer in a wall-bounded pin matrix, 2010 14th International Heat Transfer Conference, IHTC 14 Volume 2, 2010, Pages 807-815 2010 14th International Heat Transfer Conference, IHTC 14; Washington, DC; United States; 8 August 2010 through 13 August 2010.

https://doi.org/10.1115/IHTC14-23205 
[2]. Delibra, G., Borello, D., Hanjalic, K., Rispoli, F., Hybrid LES/RANS of internal flows: A case for more advanced RANS, Notes on Numerical Fluid Mechanics and Multidisciplinary Design Volume 130, 2015, Pages 19-35.

https://doi.org/10.1007/978-3-319-15141-0_2

[3]. Oztop Hakan F., Khudheyer S. Mushatet, and İlker Yılmaz. "Analysis of turbulent flow and heat transfer over a double forward-facing step with obstacles." International Communications in Heat and Mass Transfer 39.9 (2012): 1395-1403. https://doi.org/10.1016/j.icheatmasstransfer.2012.07.011

[4]. H. G. Weller, G. Tabor, H. Jasak, C. Fureby, A tensorial approach to computational continuum mechanics using object-oriented techniques, COMPUTERS IN PHYSICS, VOL. 12, NO. 6, NOV/DEC 1998.

https://doi.org/10.1063/1.168744

[5]. Delibra, G., Borello, D., Hanjalić, K., Rispoli, F., URANS of flow and endwall heat transfer in a pinned passage relevant to gas-turbine blade cooling, International Journal of Heat and Fluid Flow Volume 30, Issue 3, June 2009, Pages 549-560. https://doi.org/10.1016/j.ijheatfluidflow.2009.03.015

[6]. Launder, B. E. and Sharma, B. I. (1974), "Application of the Energy-Dissipation Model of Turbulence to the Calculation of Flow Near a Spinning Disc", Letters in Heat and Mass Transfer, Vol. 1, No. 2, pp. 131-138.

https://doi.org/10.1016/0094-4548(74)90150-7

[7]. Goodfellow Ian, Yoshua Bengio, and Aaron Courville. "Deep Learning". MIT press, 2016.

http://www.deeplearningbook.org

[8]. Wu JL, Xiao H, Paterson EG. 2018b. Physics-informed machine learning approach for augmenting turbulence models: a comprehensive framework. Phys. Rev. Fluids 3: 074602.

https://doi.org/10.1103/PhysRevFluids.3.074602

[9]. G. Angelini, A. Corsini, G. Delibra, M. Giovannelli, Identification of Losses in Turbomachinery with Machine Learning, GT2020-15337.

[10]. A. Corsini, G. Delibra, M. Giovannelli, S. Traldi, Machine Learnt Synthetic Turbulence for LES Inflow Conditions, GT2020-15338.

[11]. A. Corsini, G. Delibra, M. Giovannelli, G. Lucherini, S. Minotti, S. Rossin, L. Tieghi, Prediction of Ventilation Effectiveness for LM9000 Package With Machine Learning, GT2020-14916.

[12]. Chen, T., \& Guestrin, C. (2016). XGBoost: A Scalable Tree Boosting System. In Proceedings of the 22nd ACM SIGKDD International Conference on Knowledge Discovery and Data Mining (pp. 785-794). New York, NY, USA: ACM. https://doi.org/10.1145/2939672.2939785

[13]. Bergstra, J. and Bengio, Y., Random search for hyper-parameter optimization, The Journal of Machine Learning Research (2012).

https://jmlr.org/papers/volume13/bergstra12a/bergstra12a.pdf 